\title{
Chapter 2 \\ A High-Quality Finger Vein Dataset Collected Using a Custom-Designed Capture Device
}

\section{Raymond Veldhuis, Luuk Spreeuwers, Bram Ton and Sjoerd Rozendal}

\begin{abstract}
High-quality finger vein datasets available for the research community are still relatively scarce; therefore, we collected a set of finger vein images of high resolution and a known pixel density. Furthermore, this is the first dataset which contains the age, gender and handedness of the participating data subjects as metadata. This dataset has been collected using a custom-designed biometric capture device. The various aspects of designing this biometric capture device are addressed in this chapter. New insights and continuing work on the design of better capture devices have led to novel ideas which are presented in this chapter. To justify the importance of this dataset, performance figures in terms of EER of several well-established algorithms using this dataset and an existing dataset are compared side by side.
\end{abstract}

Keywords Finger vein capture device $\cdot$ Finger vein data set $\cdot 3 \mathrm{D}$ finger vein reconstruction

\subsection{Introduction}

The vascular or vein pattern of the finger is advertised as a promising new biometric characteristic. Biometric recognition based on finger vein patterns is characterised by very low error rates, good presentation attack resistance and a user convenience that is equivalent to that of fingerprint recognition. Though this new form of biometrics is already commercially deployed, it still lacks a strong scientific base. This is due to

R. Veldhuis $\cdot$ L. Spreeuwers $(\varangle) \cdot$ B. Ton

Data Science Group, Faculty of EEMCS, University of Twente, Enschede, The Netherlands

e-mail: 1.j.spreeuwers@utwente.nl

R. Veldhuis

e-mail: r.n.j.veldhuis@utwente.nl

B. Ton

e-mail: b.t.ton@alumnus.utwente.nl

S. Rozendal

University of Twente, Enschede, Netherlands

e-mail: s.p.rozendal@student.utwente.nl

(C) The Author(s) 2020

A. Uhl et al. (eds.), Handbook of Vascular Biometrics, Advances in Computer Vision and Pattern Recognition, https://doi.org/10.1007/978-3-030-27731-4_2 
industrial protectiveness, which restricts the ability to verify claimed performances. In order to compare existing algorithms, a standardised testing method is needed and more datasets should be made available to researchers.

In order to stimulate the academic research on vascular pattern recognition, this chapter will present a finger vascular pattern dataset which has recently been made available to other researchers [17]. The presented dataset is unique in its kind as it provides high-resolution images together with demographics about the data subjects. Another contribution of this chapter is the performance verification of several published algorithms using both the newly collected dataset and an existing dataset collected by the Peking University [12].

Our dataset has been collected using a custom-designed capture device. The various aspects of designing this capture device are also covered in this chapter.

In the remainder of this chapter, first a brief overview is provided of finger vein acquisition techniques and systems in Sect.2.2. Next, the custom-designed capture device is described in detail (Sect. 2.3), followed by the dataset (Sect. 2.4). In Sect. 2.5, results of various finger vein recognition algorithms on the database are presented. Section 2.6 presents the next-generation finger vein scanner currently under development at the University of Twente: a more compact design with 3D capabilities and other enhancements. Section 2.7 presents conclusions and in Sect. 2.8 future work is described.

\subsection{Overview of Finger Vein Acquisition Systems}

\subsubsection{Types of Sensors}

We first briefly summarise the different types of sensors for finger vein recognition and then present our own design. Devices that capture the vascular pattern inside a finger are based on the fact that the haemoglobin inside the veins has a higher absorption of Near-Infrared Light (NIR light) than the surrounding tissue. This means that the vascular pattern inside a finger can be captured by a device that is sensitive to NIR light. The veins have to be made visible with NIR light, but there are multiple possibilities to illuminate the finger. The main types that are found in existing devices are shown in Fig. 2.1.

The illumination with the light reflection method is on the same side as the camera. This allows the device to be more compact. During operation, the user of the device can still see his finger. The disadvantage of this method is that the image sensor mainly captures the reflected light from the surface of the finger, because the light shallowly penetrates the skin. Hence, this method gives images with low contrast between tissue and veins. The light transmission method does deliver high-contrast vascular pattern images, because the light passes through the finger and no reflections of the surface are captured. The illumination is at the other side of the finger relative to the camera. The disadvantage of this method is that the user has to put his finger into the device such that he cannot see his finger anymore, which can cause discomfort. The third illumination type is side lighting method. This method still allows an open 


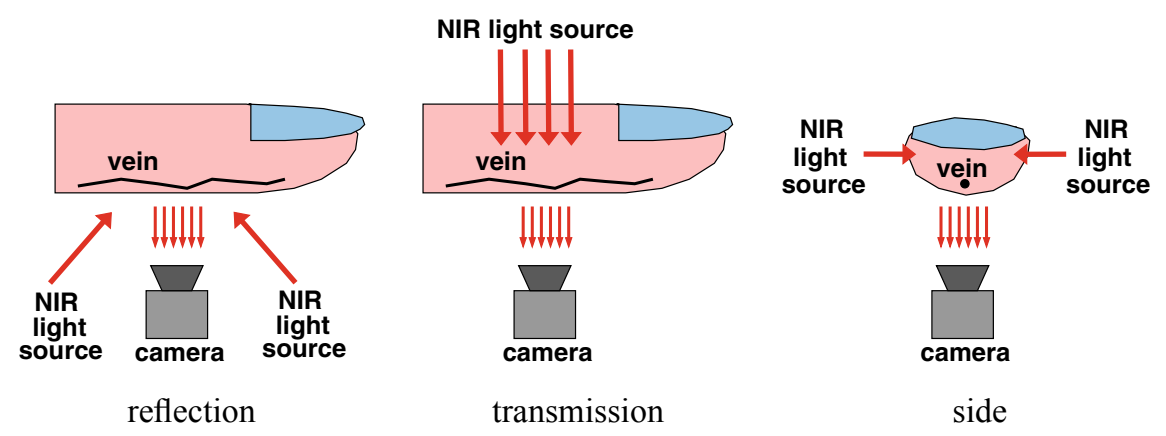

Fig. 2.1 Reflection, transmission and side illumination acquisition

device such that the user can see his finger. The light sources are placed on either one side or both sides of the finger. NIR light goes through the sides of the finger and scatters there, before it is captured by the image sensor. This method does allow for high-contrast images. However, the sides of the finger are overexposed in the images.

Some examples of commercially available sensors and sensors developed by academics are presented in Sects. 2.2.2 and 2.2.3. For a more complete overview, please refer to Chap. 3 of this book.

\subsubsection{Commercial Sensors}

There are several devices on the market for vascular pattern recognition. The market leader in finger vein capture devices is Hitachi. They have developed multiple systems that are capable of capturing finger vein images using light transmission or side illumination. Hitachi claims that it has a False Non-Match Rate (FNMR) of $0.01 \%$ at a False Match Rate (FMR) of $0.0001 \%$ [3, 4]. However, it is hard to verify these claims, because the devices and image data are not accessible.

Another company that builds finger vein capture devices is Mofiria, a daughter company of Sony. This company also produces various devices among which one using light transmission, but where the finger is placed sideways on the sensor. They claim an FNMR of $0.1 \%$ at an FMR of $0.0001 \%$ [15], but again these are closed devices and data are not accessible.

\subsubsection{Sensors Developed by Academics}

At several universities, research into finger vein recognition is performed and acquisition devices were developed. Examples are the finger vein scanner devices developed by the Civil Aviation University of China [21] and the University of Electronic Science and Technology [9]. The latter device also has the capability of making $3 \mathrm{D}$ recordings of finger veins. A more recent sensor, developed at the Norwegian 
Biometrics Laboratory (NBL), allows simultaneous capturing of both finger vein patterns and fingerprints [13]. This is a closed sensor, and the user has to place his finger through a hole inside the device.

The device developed at the University of Twente, which is described in the subsequent sections, is also an example of this group of finger vein acquisition devices. The huge advantage of these devices, developed by academics, is that they are usually open devices, the image data is accessible and datasets are made available to the research community. This enables us to evaluate and compare various methods for finger vein recognition.

\subsection{University of Twente Finger Vein Capture Device}

A custom transillumination device type has been designed to capture the finger vascular pattern $[18,19]$. This type of capture device has been chosen for its simplicity, robustness and the fact that external light interferences have little influence on the captured images. A downside of this type of capture device is the reduced user convenience because the finger is partially obscured during the capture process. All finger vascular pattern capture devices are based on the fact that blood has a higher absorbency than surrounding tissue in the near-infrared spectrum. A schematic cross section of the capture device can be seen in Fig. 2.2. The USB lightbox is responsible for regulating the individual LED intensities and is encapsulated in the capture device for the ease of portability. The overview also shows the slanted mirror indicated in green and the top plate containing the eight LEDs. The total length of the realised capture device is $50 \mathrm{~cm}$, and the maximum height is $15 \mathrm{~cm}$.

The constructed capture device consists of three main components: a light source, a camera and a mirror. These components will be described briefly in the successive paragraphs.

Light source This the most important part of the capture device since it determines the intensity of the captured image. Eight SFH4550 near-infrared LEDs produced by Osram with a wavelength of $850 \mathrm{~nm}$ are used to transilluminate the finger. This LED type has been chosen because it has a small angle of half intensity, which means more power can be directed into the finger. Each individual LED intensity is regulated using a simple control loop in such a way that a uniform intensity along

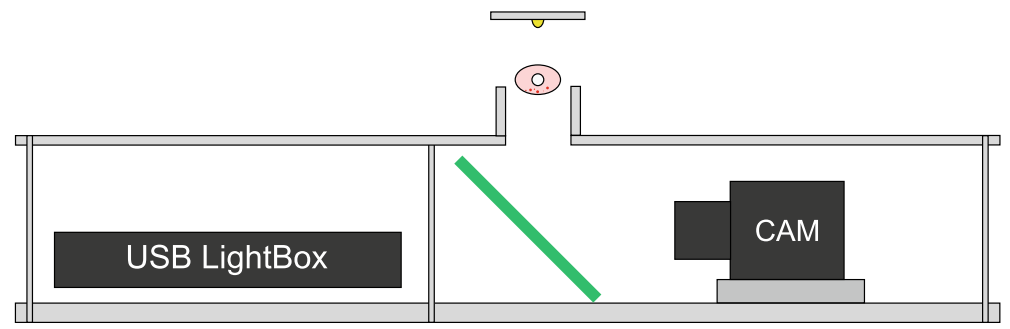

Fig. 2.2 Schematic cross section of the capture device 
the finger is obtained in the captured image. This control loop is also necessary to cope with varying thicknesses along the finger and between various biometric data subjects. The benefit of this simple control loop can be seen in Fig. 2.3. It clearly shows the over- and underexposure in the non-regulated case.

Camera The camera used to capture the images is a BCi5 monochrome CMOS camera with firewire interface produced by C-Cam technologies. The camera has been fitted with a Pentax H1214-M machine vision lens with a focal length of $12 \mathrm{~mm}$. This lens is fitted with a B+W 093 infrared filter which has a cutoff wavelength of $930 \mathrm{~nm}$. The filter is used to block out any interfering visible light. The camera is used in 8-bit mode with a resolution of $1280 \times 1024$ pixels.

Mirror To minimise the height of the capture device, a mirror is used so the camera can be placed horizontally. An NT41-405 first surface mirror produced by Edmund Optics has been used for this purpose. The reason for choosing a first surface mirror is to avoid distortions in the captured image. A conventional mirror has its

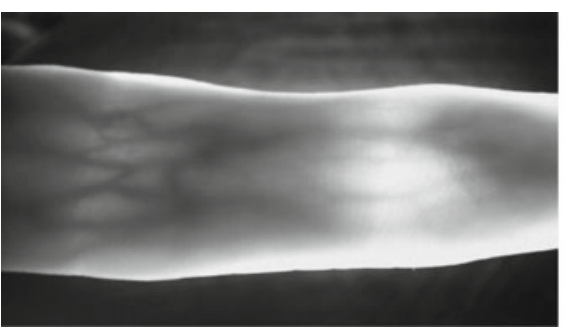

(a) Eight equal LED intensities

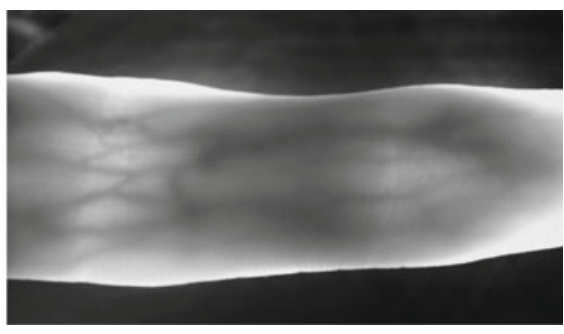

(b) LED intensities regulated by control loop

Fig. 2.3 Benefit of the control loop to adjust the individual LED intensities

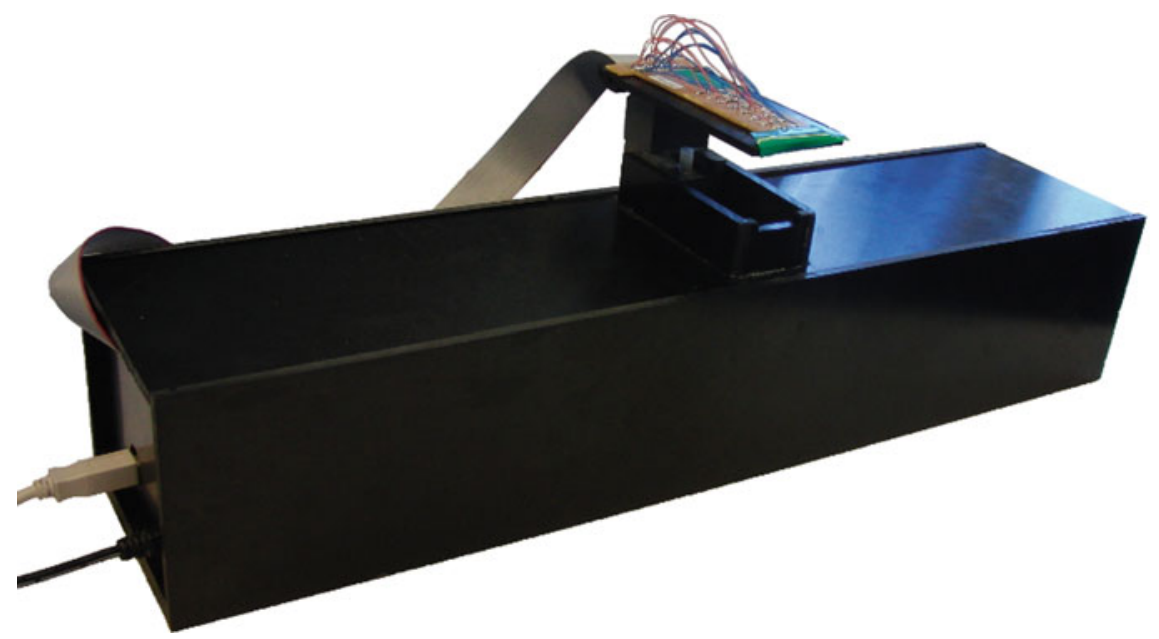

Fig. 2.4 Realised finger vascular pattern capture device 
reflective layer protected by glass. The refractive indices of glass and air differ which means distortions will occur in the captured image. The final constructed capture device can be seen in Fig. 2.4.

\subsection{Description of Dataset}

The University of Twente Finger Vein Pattern (UTVP) dataset contains 1440 finger vascular pattern images in total which have been collected from 60 volunteering subjects at our university during the 2011-2012 academic year. Images were captured in two identical sessions with an average time-lapse of 15 days. For each data subject, the vascular pattern of the index, ring and middle finger of both hands has been collected twice at each session. This means that each individual finger has been captured four times in total. The captured images have a resolution of $672 \times 380$ pixels and have a pixel density of 126 pixels per centimetre $(\mathrm{ppcm})$. The images are stored using the lossless 8-bit greyscale Portable Network Graphics (PNG) format. The percentage of male data subjects was $73 \%$, and the percentage of right-handed data subjects was $87 \%$. The dataset represents a young population with $82 \%$ of the data subjects falling in the age range of 19-30, and the remaining data subjects were older than this. A set of sample images from the collected dataset can be seen in Fig. 2.5. The quality of the collected images varies among biometric capture subjects, but the variation in quality of the images from the same biometric capture subject is small.

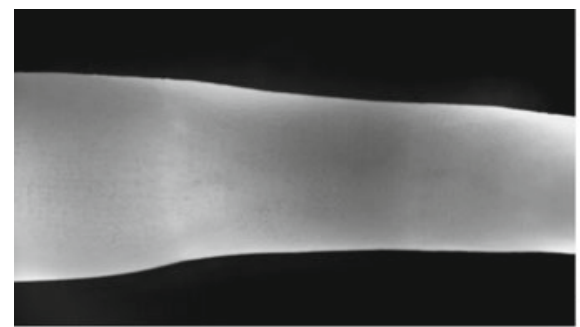

(a) Female, age 24

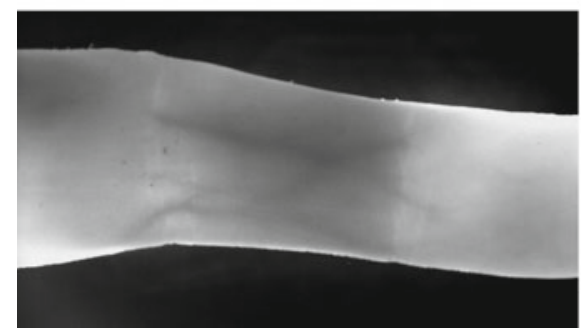

(c) Male, age 20

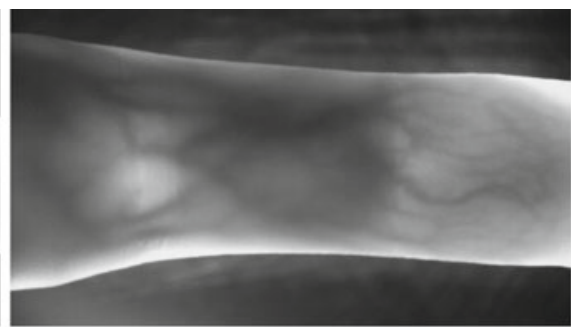

(b) Male, age 32

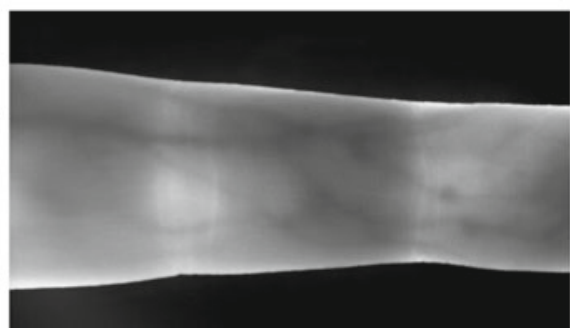

(d) Female, age 31

Fig. 2.5 Sample images of the left-hand ring finger from the collected dataset 
The width of the visible blood vessels ranges from 4-20 pixels which, using a pixel density of 126 pixels per centimetre, corresponds to vessel widths of approximately $0.3-1.6 \mathrm{~mm}$. The pixel density was determined by placing a piece of flat graph paper at exactly the same position as the finger and counting the number of pixels per centimetre in the recorded image. This resulted in a pixel density of 126 pixels per centimetre.

The UTVP dataset is available from the University of Twente by completing an online download request and license agreement, see [17].

\subsection{Results}

\subsubsection{Performance Analysis}

To illustrate and rank the quality of the collected dataset, the performance of a few published algorithms was evaluated. These algorithms have been applied to our collected dataset and the V4 finger vein database from the Peking University [12] which has been used as a reference. The performance of the algorithms is measured in terms of Equal Error Rate (EER). The experiments also investigate the merit of Adaptive Histogram Equalisation (AHE) as a preprocessing step. Each directory of the Peking dataset contains between four and eight images of the same finger. For the experiments only directories containing exactly eight images have been used, this accounts for 153 directories out of the available 200 directories. For this dataset, it is not known which fingers belong to the same subject.

For both datasets, $10 \%$ of the fingers have been used for tuning the various parameters of the algorithms. For the Peking dataset, the valid directories are sorted ascending by filename and the first $10 \%$ are used for parameter tuning. For our dataset, $10 \%$ of the fingers have been selected by taking the first finger of the first data subject, the second finger of the second data subject ... the first finger of the seventh data subject. This method of selecting the training set has been chosen to get a larger variation in the quality of the vascular pattern images. The other $90 \%$ of both datasets have been used to determine the actual performance of the algorithms.

The exact number comparison trials done for both the parameter tuning and the actual determination of the performance are given in Table 2.1.

For all of these experiments, fingers were treated as identical individual biometric samples, for example, left-hand index fingers were compared with right-hand middle fingers. Two performance experiments are done per dataset, one with and one without adaptive histogram equalisation as preprocessing step. This preprocessing step is done using MATLAB's adapthisteq ( ) function with the default parameters' set. The effect of applying an adaptive histogram equalisation to a vascular pattern image can be seen in Fig. 2.6.

To ensure that only image regions containing finger are compared with each other a binary mask is used. This mask is created by first determining the edges of the 
Table 2.1 Number of mated and non-mated comparison trials performed

\begin{tabular}{|c|c|c|c|}
\hline Dataset & \# fingers & Mated & Non-mated \\
\hline \multicolumn{4}{|c|}{ Parameter tuning } \\
\hline Peking & 15 & 420 & 6720 \\
\hline UTFVP & 35 & 210 & 9520 \\
\hline \multicolumn{4}{|c|}{ Actual performance experiment } \\
\hline Peking & 138 & 3864 & 604,992 \\
\hline UTFVP & 325 & 1950 & 842,400 \\
\hline
\end{tabular}

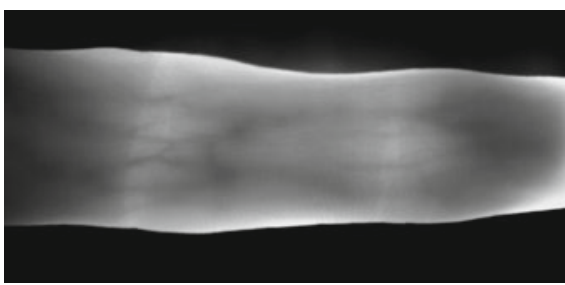

(a) Original image

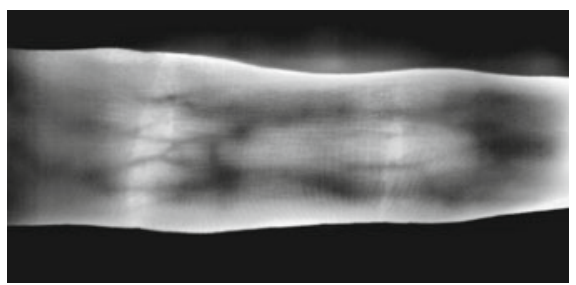

(b) Adaptive Histogram Equalisation

Fig. 2.6 Effect of Adaptive Histogram Equalisation

finger in the image using the method described by Lee et al. [8] and then filling in the area between these edges.

The edges detected in the previous step are used to normalise the image using the method described by Huang et al. [5]. This method tries to estimate a rotation and a translation based on the detected finger edges. After these parameters have been estimated, they are used to define an affine image transformation which aligns the finger to the centre of the image. This affine transformation is also applied to the binary mask.

The output of each of the algorithms, except the normalised cross-correlation, is a binary template indicating the position of a blood vessel. Two binary templates are compared with each other by using the method described by Miura et al. [10]. An incidental side effect of using the binary finger region mask is that the shape of the finger is also indirectly taken into account when comparing two templates.

The final verification results are shown in Table 2.2 which indicates that our dataset performs significantly better in all cases and that adaptive histogram equalisation is beneficial in most of the cases. The results presented here have been independently replicated by Vanoni et al. [20].

The two methods proposed by Miura et al. have been tested by other researchers using their own collected datasets. One of them is Huang et al. [5] who has achieved an EER of $2.8 \%$ for the maximum curvature method and an EER of $5 \%$ for the repeated line tracking method. Another one is Choi et al. [2] who have achieved an EER of $3.6 \%$ for the maximum curvature method. The last one is Kumar and Zhou [7] who achieved an EER of $8.3 \%$ for the repeated line tracking method and 
Table 2.2 Performance expressed in terms of EER (\%) of several algorithms for both datasets, both with and without Adaptive Histogram Equalisation (AHE) as a preprocessing step

\begin{tabular}{|c|c|c|c|c|c|c|}
\hline & \multicolumn{6}{|l|}{ EER (\%) } \\
\hline & \multirow[t]{2}{*}{ Original paper } & \multirow[t]{2}{*}{ Best reported } & \multicolumn{2}{|l|}{ Peking } & \multicolumn{2}{|l|}{ UTFVP } \\
\hline & & & No AHE & With AHE & No AHE & With AHE \\
\hline $\begin{array}{l}\text { Normalised } \\
\text { cross- } \\
\text { correlation }^{\mathrm{a}}\end{array}$ & 0.0 & - & 14.7 & 9.8 & 3.1 & 1.9 \\
\hline $\begin{array}{l}\text { Maximum } \\
\text { curvature }^{b}\end{array}$ & 0.0 & $2.7^{\mathrm{e}}$ & 1.2 & 1.3 & 0.4 & 0.4 \\
\hline $\begin{array}{l}\text { Repeated } \\
\text { line } \\
\text { tracking }\end{array}$ & 0.2 & $5.0^{\mathrm{f}}$ & 6.8 & 5.9 & 0.9 & 1.2 \\
\hline $\begin{array}{l}\text { Principal } \\
\text { curvature }^{\mathrm{d}}\end{array}$ & 0.4 & - & 2.7 & 2.2 & 0.8 & 0.4 \\
\hline $\begin{array}{l}\text { Wide line } \\
\text { detector }^{\text {e }}\end{array}$ & 0.9 & - & 4.7 & 2.7 & 1.5 & 0.9 \\
\hline
\end{tabular}

${ }^{\text {a }}$ See [6]

${ }^{\mathrm{b}}$ See [11]

${ }^{\mathrm{c}}$ See $[10]$

${ }^{\mathrm{d}}$ See [2]

${ }^{\mathrm{e}}$ See [5]

${ }^{\mathrm{f}}$ See [7]

achieved an EER of $2.7 \%$ for the maximum curvature method. The mentioned EERs from Kumar and Zhou are the average EER of the middle and index fingers. The best reported performance figures for these two methods are mentioned in Table 2.2 as well. Our MATLAB implementation of these algorithms can be found in [16].

\subsection{Next-Generation Finger Vein Scanner}

\subsubsection{Overview}

Since the design of the described finger vein scanner, we developed a second version of the finger vein scanner with new capabilities, see Fig. 2.7 [14]. The scanner is much more compact and is built using cheaper components: it uses Raspberry Pi processing boards and cameras. This new scanner has been designed in such a way as to support further research in various ways. It supports multiple NIR LED strips that can be positioned in a semicircle from $0^{\circ}$ to $180^{\circ}$. It also supports three cameras, thus allowing for 3D finger vein reconstruction. Currently, we are investigating optimal illumination and settings of the cameras and 3D finger vein reconstruction. 

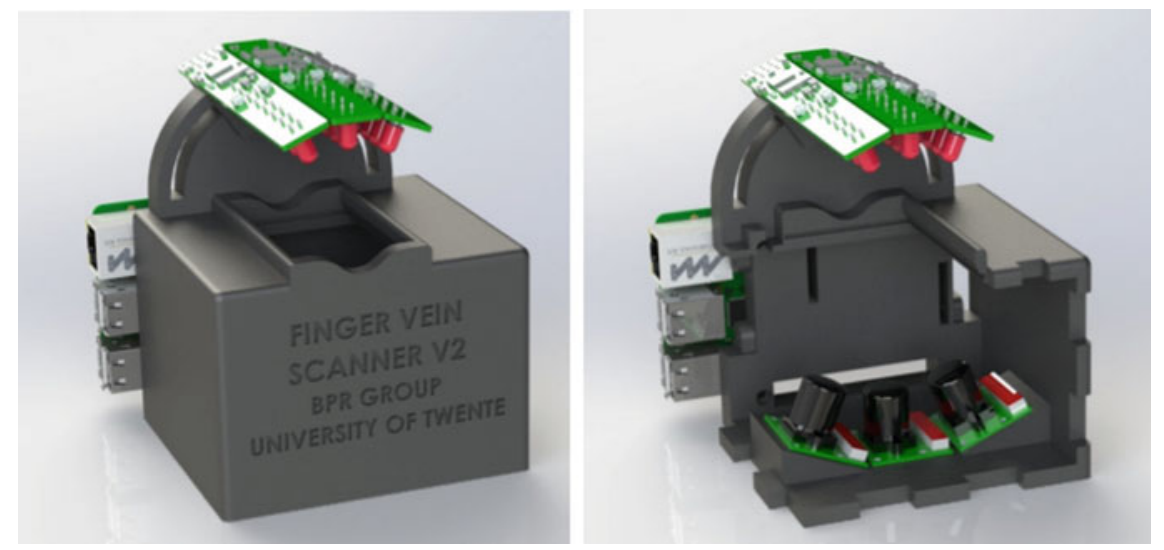

Fig. 2.7 Second-generation finger vein scanner of the University of Twente. It has three cameras for $3 \mathrm{D}$ recordings and multiple adjustable LED strips

\subsubsection{Illumination Control}

The setup with multiple LED strips that can be rotated up to $180^{\circ}$ allows for various illumination methods. It supports both transmission and side illumination. Reflection is not supported, however. Care was taken to position the LED strip with respect to the finger position and the opening for the finger with below it the infrared filter, such that as little as possible infrared light can "leak" around the finger. The new setup inherited the advanced control over the intensity of each individual LED from the previous version of the scanner, enabling a more homogeneous illumination and adjustment to the properties of the finger (e.g. thick and thin fingers). In Fig. 2.8, a comparison is made between images recorded using the first- and second-generation finger vein scanner of the University of Twente. The images of the new scanner show much less overexposure at the boundaries of the fingers.

We are currently investigating various ways to optimise illumination ranging from illumination from different angles and multiple LED strips to refined control of the LED intensities and combination of multiple images with different illumination.

\subsubsection{D Reconstruction}

The advantage of $3 \mathrm{D}$ recordings is that if fingers are slightly rotated, causing a deformation of the finger vein pattern, this deformation can be compensated for. Another possibility is direct comparison of 3D finger vein patterns. Using the three cameras in the new scanner, we used stereo reconstruction to recover the $3 \mathrm{D}$ vein patterns. A preliminary result of 3D finger vein reconstruction using this secondgeneration finger vein scanner is shown in Fig. 2.9 [1]. 

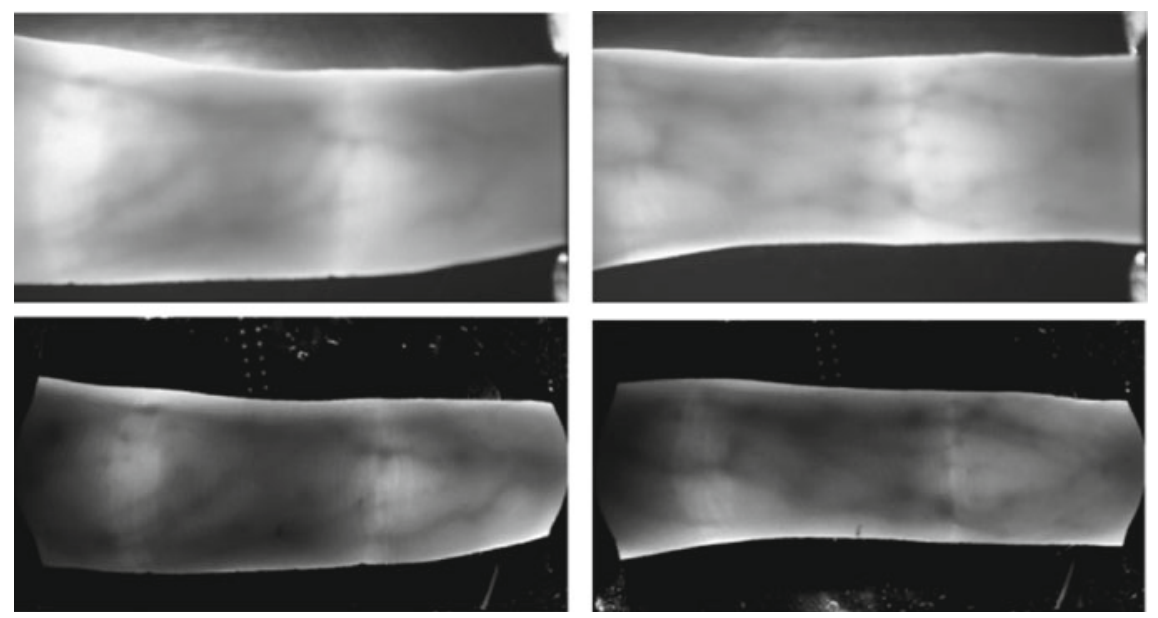

Fig. 2.8 Comparison between images of the same fingers captured by the first (top)- and second (bottom)-generation finger vein scanners. The images captured by the new scanner show less overexposure near the boundaries of the fingers

Fig. 2.9 Preliminary 3D

finger vein reconstruction using new vein scanner

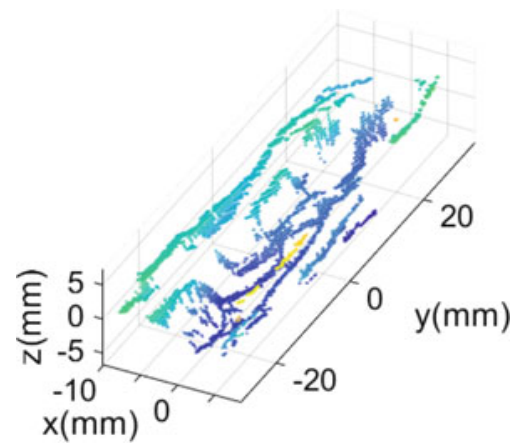

Careful observation shows that the veins at the sides of the fingers are somewhat above the veins in the middle of the finger, i.e. they follow the curvature of the surface of the finger. This is to be expected, because only the veins at the surface of the finger can be visualised using this technique.

\subsection{Conclusions}

A finger vascular pattern dataset containing 1440 high-quality images is presented to the research community. Despite the low number of 60 data subjects which participated, the major contribution of this dataset is the addition of demographic data such as gender, age, and handedness. Another contribution is the high quality of the captured images and the known pixel density of the images. Furthermore, the data 
is collected in two identical sessions with a time lapse of approximately 2 weeks. Because of the high quality of the captured images, our dataset can pave the way for the research of high-security cooperative applications. The performance evaluation using existing algorithms has shown that equal error rates down to $0.4 \%$ can be achieved by using our dataset.

\subsection{Future Work}

The use of the vascular pattern of the finger as a biometric is still not as mature as other biometric traits such as $2 \mathrm{D}$ face recognition. To reach an equal maturity, more research is needed.

Future research should include the collection of larger datasets including 3D data, together with demographic data of the data subjects. These larger datasets will enable researchers to report performance figures with a higher confidence. It will also enable the research of factors such as age, gender and ethnicity on the performance. The research community would also greatly benefit from standardised testing methods and datasets.

The biometric performance can further be improved by fusing other finger traits such as traditional fingerprints, the crease pattern of the finger and the shape of the finger. An advantage of finger shape is that it is already present in the captured image.

The current control loop which adjusts the LED intensities is still rather crude and leaves space for further improvements in terms of speed and image intensity uniformity. Preliminary results have shown that the relation between the intensity in the captured image and the intensity of the LEDs is as good as linear.

Finally, 3D scanning techniques allow compensation of distortions of the finger vein pattern caused by rotation of the finger. Also, direct $3 \mathrm{D}$ finger vein comparison is an interesting subject for further research.

\section{References}

1. Bunda S (2018) 3D point cloud reconstruction based on the finger vascular pattern. Bachelor's thesis, University of Twente, Netherlands

2. Choi J, Song W, Kim T, Lee S, Kim H (2009) Finger vein extraction using gradient normalization and principal curvature. In: Niel KS, Fofi D (eds) Image processing: machine vision applications II, SPIE-IS\&T Electronic Imaging, vol 7251. SPIE. https://doi.org/10.1117/12.810458

3. Hitachi Ltd. (2018) Finger vein reader. http://www.hitachi.co.jp/products/it/veinid/global/ products/embeddeddevicesr.html

4. Hitachi Ltd. (2018) USB finger vein biometric authentication unit. http://www.hitachi.co.jp/ products/it/veinid/global/products/embeddeddevicesu.html

5. Huang B, Dai Y, Li R, Tang D, Li W (2010) Finger-vein authentication based on wide line detector and pattern normalization. In: 20th International conference on pattern recognition, pp 1269-1272. https://doi.org/10.1109/ICPR.2010.316

6. Kono M, Ueki H, Umemura S (2002) Near-infrared finger vein patterns for personal identification. Appl Opt 41(35):7429-7436. https://doi.org/10.1364/AO.41.007429 
7. Kumar A, Zhou Y (2012) Human identification using finger images. IEEE Trans Image Process 21(4):2228-2244. https://doi.org/10.1109/TIP.2011.2171697

8. Lee E, Lee H, Park K (2009) Finger vein recognition using minutia-based alignment and local binary pattern-based feature extraction. Int J Imaging Syst Technol 19(3):179-186. https://doi. org/10.1002/ima.20193

9. Ma Z, Fang L, Duan J, Xie S, Wang Z (2016) Personal identification based on finger vein and contour point clouds matching. In: IEEE International conference on mechatronics and automation, pp 1983-1988

10. Miura N, Nagasaka A, Miyatake T (2004) Feature extraction of finger vein patterns based on iterative line tracking and its application to personal identification. Syst Comput Jpn 35:61-71. https://doi.org/10.1002/scj.v35:7

11. Miura N, Nagasaka A, Miyatake T (2005) Extraction of finger-vein patterns using maximum curvature points in image profiles. In: IAPR conference on machine vision applications, vol 9, pp 347-350. http://b2.cvl.iis.u-tokyo.ac.jp/mva/proceedings/CommemorativeDVD/2005/ papers/2005347.pdf

12. Peking University (2013) PKU finger vein database. http://rate.pku.edu.cn

13. Raghavendra R, Raja KB, Surbiryala J, Busch C (2014) A low-cost multimodal biometric sensor to capture finger vein and fingerprint. In: IEEE International joint conference on biometrics, pp 1-7. https://doi.org/10.1109/BTAS.2014.6996225

14. Rozendal S (2017) Redesign of a finger vein scanner. Bachelor's thesis, University of Twente, Netherlands

15. Sony Corporation (2009) Sony develops compact sized, high speed, high accuracy finger vein authentication technology dubbed "mofiria". https://www.sony.net/SonyInfo/News/Press/ 200902/09-016E/index.html

16. Ton B $(2012,2017)$ MATLAB implementation of Miura et al. vein extraction methods. University of Twente, Netherlands. https://www.mathworks.com/matlabcentral/fileexchange/35716miura-et-al-vein-extraction-methods

17. Ton B, Veldhuis R (2012) University of Twente Finger Vascular Pattern (UTFVP) dataset. https://www.utwente.nl/en/eemcs/ds/downloads/utfvp/

18. Ton BT (2012) Vascular pattern of the finger: biometric of the future? sensor design, data collection and performance verification. Master's thesis, University of Twente

19. Ton BT, Veldhuis RNJ (2013) A high quality finger vascular pattern dataset collected using a custom designed capturing device. In: 2013 International conference on biometrics (ICB), pp 1-5. https://doi.org/10.1109/ICB.2013.6612966

20. Vanoni M, Tome P, Shafey LE, Marcel S (2014) Cross-database evaluation using an open finger vein sensor. In: Workshop on biometric measurements and systems for security and medical applications, pp 30-35. https://doi.org/10.1109/BIOMS.2014.6951532

21. Yang J, Shi Y, Yang J (2011) Personal identification based on finger-vein features. Comput Hum Behav 27:1565-1570

Open Access This chapter is licensed under the terms of the Creative Commons Attribution 4.0 International License (http://creativecommons.org/licenses/by/4.0/), which permits use, sharing, adaptation, distribution and reproduction in any medium or format, as long as you give appropriate credit to the original author(s) and the source, provide a link to the Creative Commons license and indicate if changes were made.

The images or other third party material in this chapter are included in the chapter's Creative Commons license, unless indicated otherwise in a credit line to the material. If material is not included in the chapter's Creative Commons license and your intended use is not permitted by statutory regulation or exceeds the permitted use, you will need to obtain permission directly from the copyright holder. 